\title{
An Urgent Demand for Novel, Safe Cell Sources for Musculoskeletal Regeneration
}

\author{
Xiaoxiao Pang ${ }^{1,2}$, Nathaniel Burdekin ${ }^{3}$, Chenshuang $\mathrm{Li}^{1}$, Zhong Zheng ${ }^{1 *}$ \\ ${ }^{1}$ Division of Growth and Development, School of Dentistry, University of California, \\ Los Angeles, CA 90095, USA; \\ ${ }^{2}$ Chongqing Key Laboratory of Oral Diseases and Biomedical Sciences, \\ Stomatological Hospital of Chongqing Medical University, Chongqing 401147, China; \\ ${ }^{3}$ Department of Chemistry and Biochemistry, University of California, Los Angeles, \\ CA 90095, USA.
}

*Corresponding Author: Zhong Zheng. Email: zzheng@dentistry.ucla.edu.

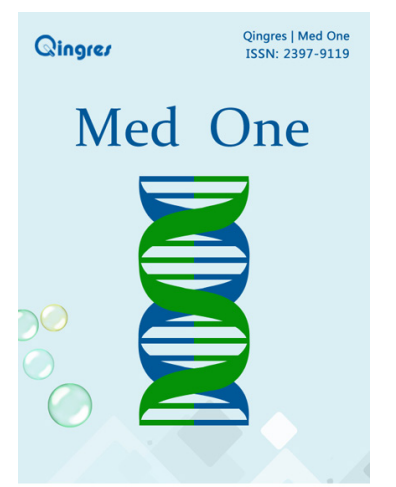

https://mo.qingres.com

\section{GOPEN ACCESS}

DOI: $10.20900 / m o .20180010$

Received: September 12, 2018

Accepted: October 15, 2018

Published: October 19, 2018

Copyright: $\odot 2018$ by the authors, Licensee Qingres, London, United Kingdom. This is an open access article distributed under the terms and conditions of the Creative Commons Attribution License (CC BY 4.0, https://creativecommons.org/licenses/by/4.0/).

\begin{abstract}
Musculoskeletal disorders affect a significant proportion of people, especially among the aging population. In comparison with traditional treatment, multi/pluripotent cell-based therapies exhibit better performance in their ability to induce the regeneration of damaged musculoskeletal tissues and are regarded as a promising therapeutic method for the purpose of musculoskeletal regeneration. This minireview comprises a brief overview of recently investigated multi/pluripotent cells and their effectiveness in musculoskeletal regeneration. Furthermore, advantages, drawbacks and future perspectives on different multi/pluripotent cells are concisely addressed, which point to an emergent demand for a novel regenerative cell source that can be safely used for musculoskeletal tissue regeneration.
\end{abstract}

Keywords: multi/pluripotent cells; tumorigenesis; musculoskeletal regeneration; reprogramming

\section{INTRODUCTION}

The musculoskeletal system consists of muscles, bones, cartilage, tendons, ligaments, joints and other connective tissues, which give form, support, stability, and movement to the body. Diseases of the musculoskeletal system are the most common functional disorders or motion discordances, which can affect many different parts of the body. Musculoskeletal disorders are the second leading cause of disability and have increasingly become a global healthcare issue ${ }^{[1]}$. More than half of people over the age of 18 in the US suffer from a 
musculoskeletal disorder ${ }^{[2]}$. These disorders account for more than $\$ 200$ billion in medical expenses on an annual basis ${ }^{[2]}$. A variety of procedures have been conducted to enhance the repair or regeneration of damaged musculoskeletal tissues, but healing is imperfect in many cases. This is especially true in extensive-size defects where the endogenous progenitors are unable to restore tissue integrity and function. Thanks to advances in stem cell biology and cell reprogramming technology, a diversity of multipotent and pluripotent cells have been investigated for the purpose of musculoskeletal regeneration, which mostly overcome the difficulties associated with traditional treatments. However, several drawbacks including donor site damage, deficient cell availability, poor survivability, restricted proliferation, limited spreading, and dedifferentiation must be overcome before these cells can be officially used in clinical therapy ${ }^{[3]}$.

\section{DIFFERENT STEM CELLS IN MUSCULOSKELETAL REGENERATION}

\subsection{Adult mesenchymal stem cells}

For decades, a number of multipotent cell sources have been explored for the purpose of musculoskeletal regeneration. The most notable among these cells are mesenchymal stem cells (MSCs). Adult MSCs can be isolated from a variety of tissues including bone marrow, adipose tissue, perichondrium, cartilage, tendon, muscle, skin, dental pulp, gut, liver, salivary glands, etc. However, isolating procedures are generally invasive and painful, and often supply an insufficient yield of MSCs, resulting in the need for long-term cultivation ${ }^{[4]}$, which can jeopardize the differentiation potential of adult MSCs ${ }^{[5]}$ and induce malignant transformation ${ }^{[6]}$. In addition, as the age of the donor increases, the cells' capability to proliferate and differentiate is significantly reduced ${ }^{[7,8]}$. This raises more serious concerns regarding the prospect of obtaining a sufficient quantity of functional autogenous MSCs from elderly patients - the population with the highest demand for cell-based regenerative medicine. It is also worth noting that some researchers remain skeptical about MSCs, arguing that the effects of MSCs are exaggerated and still controversial ${ }^{[9]}$. Since the identity and biological activity of MSCs are still not fully revealed, some researchers even question the stemness of MSCs ${ }^{[9]}$. Arnorld I. Caplan at Case Western Reserve University, one of the pioneers of MSC research, suggested that endogenous MSCs derived from pericytes fabricate and secrete many bioactive factors that are strongly immunomodulatory from one side and secrete a different array of trophic factors which set-up a regenerative microenvironment from another side in the natural injury response ${ }^{[10]}$. Indeed, a recent study found that endogenous adult pericytes are not the cellular progenitors of new fibroblasts or myocytes in aging or post-injury in vivo, which challenges the current view of endogenous pericytes as multipotent tissueresident progenitors ${ }^{[11]}$. These results demonstrate that instead of directly differentiating into the target tissues, adult MSCs seem to offer regenerative benefits by producing and secreting a diverse array of bioactive soluble factors ${ }^{[10]}$. Unfortunately, these bioactive factors can also enhance the proliferation, migration, and angiogenesis of tumor cells ${ }^{[12]}$. In addition, the immunosuppressive potential of MSCs may reduce $\mathrm{T}$-cell proliferation, which, in turn, suppresses the antineoplastic response ${ }^{[12]}$. Recent studies show that human adult MSCs not only can develop chromosomal aberrations during cultivation, but also can undergo spontaneous tumorigenic transformation ${ }^{[13]}$, promote the growth of co-cultured glioblastoma multiforme cells in vitro, and support glioblastoma development in vivo ${ }^{[14]}$. Animal studies, in particular, have revealed the direct and indirect involvement of MSCs in sarcomagenesis ${ }^{[12]}$, especially in the presence of inflammatory stimulation ${ }^{[15]}$. Therefore, adult MSCs may not be a safe cell source for use in musculoskeletal regeneration, especially in an inflammatory microenvironment that is generally seen in trauma settings.

\subsection{Fetal mesenchymal stem cells}

In addition to adult MSCs, fetal MSCs isolated from umbilical cords, umbilical cord blood, amniotic membrane and amniotic fluid show similar surface marker expression, high differentiation potential, and low immunogenicity ${ }^{[16]}$. Although fetal MSCs display a remarkable aptitude for tissue regeneration, the use of fetal MSCs seems to be accompanied by more advanced technological and regulatory requirements, as well as the financial support necessary to meet these higher standards. Furthermore, the absence of standard isolation/purification methods, rejection in allogeneic use, and costly storage for future use in autologous applications pose tremendous challenges to the bench-to-bed translation of fetal MSCs ${ }^{[16]}$. Although it would be difficult to implement fetal MSC-based therapies as the standard treatment in all cases, it is possible that fetal MSCs could be a viable treatment option under specific circumstances.

\subsection{Embryonic stem cells}

Embryonic stem cells (ESCs) are another choice for musculoskeletal regeneration. These cells hold the potential to differentiate into any tissue in the body and have been investigated for their 
regenerative properties (including for osteogenic and myogenic purposes) for many years ${ }^{[17]}$. The ethics of ESC research, however, are a significant obstacle since the isolation of ESCs requires the destruction of human embryos ${ }^{[18]}$. In addition, due to the allogeneic nature of ESCs, immunogenicity in clinical use requires consideration, especially because the immunological properties of ESCs are still controversial. Findings range broadly from suggestions that ESCs can trigger an immune response to claims that ESCs are unique in their lack of immunogenicity and elicit a negligible immune response ${ }^{[19,20]}$. Importantly, the genomic instability and tumorigenic properties of ESCs make them impractical for administration in human bodies at the present stage ${ }^{[17]}$.

\subsection{Induced pluripotent stem cells}

The success of induced pluripotent stem cells (iPSCs) was a huge breakthrough for tissue regeneration. Personalized cell therapy that utilizes a patient's own cells to make iPSCs can overcome the aforementioned ethical dilemma and potential immunogenicity hindering ESCs and MSCs application. Another advantage of iPSC generation is that the isolation of starting materials does not require severely invasive or painful procedures. For instance, iPSCs can be directly reprogrammed from dermal fibroblasts-a cell source that is easily obtained and expanded from a skin biopsy ${ }^{[21]}$. In addition, the age of the donor does not affect the differentiation potential of iPSCs ${ }^{[22]}$. Unfortunately, the use of iPSCs may induce unwanted gene activation and genomic alterations due to the introduction of transcription factors (such as the Yamanaka or Thomson factors that are pivotal to embryonic development) into the genome of target somatic cells ${ }^{[23]}$. Furthermore, although multiple strategies are devoted to replacing the genomic integration procedure and oncogenic molecules, even in comparison with ESCs, iPSCs show a high tumorigenicity risk ${ }^{[23]}$, which is a nonnegligible obstruction to their administration in clinical applications.

\subsection{Multi-lineage differentiating stress enduring cells}

Multi-lineage differentiating stress enduring (MUSE) cells were first isolated from bone marrow in $2010^{[24]}$. Like ESCs and iPSCs, MUSE cells express classic pluripotency markers, although at much lower levels, and hold the potential for triploblastic differentiation from a single cell ${ }^{[25]}$. As a small population of MSCs, MUSE cells have been found throughout different mesenchymal tissues ${ }^{[26]}$. However, it is necessary for the raw material, such as bone marrow stromal cells ${ }^{[24]}$, dermal fibroblasts ${ }^{[24,25]}$ and lipoaspirate ${ }^{[27]}$, to be exposed to severe cellular stress conditions such as long-term collagenase incubation, isolation from nutrients, low temperatures, and hypoxia in order to purify the MUSE cells from the MSCs. Interestingly, in the "elite model" of iPSC generation, MUSE cells are considered to be the subpopulation of MSCs that give rise to iPSCs ${ }^{[25]}$; however, no teratoma formation was observed when MUSE cells were injected into mice throughout a 6 -month period ${ }^{[26]}$. It is worth pointing out that the mechanism governing the transition from nontumorigenic MUSE cells to tumorigenic iPSCs remains an enigma, and that the genomic stability of MUSE cells has not been fully investigated. Although MUSE cells may be a candidate for musculoskeletal tissue regeneration and stem cell therapy, there has been no direct evaluation of the efficacy or safety of MUSE cells in bone or muscle regeneration, and therefore further investigation is warranted.

\subsection{Other induced multipotent stem cells}

Inspired by previous explorations that acquired pluripotency by either transferring a somatic cell nucleus to an oocyte ${ }^{[28]}$ or fusing a somatic cell with an ESC ${ }^{[29]}$, researchers were able to successfully produce induced multipotent stem cells (iMSCs) from somatic cells via Xenopus egg extracts ${ }^{[30]}$, fish oocyte extracts ${ }^{[31]}$, ESC extracts ${ }^{[32]}$, and even carcinoma extracts ${ }^{[32]}$. Although the undefined component of these extracts makes it almost impossible to use these iMSCs in humans, the results strongly support that the microenvironment surrounding the pluripotent cells may be important in cell fate determination, including maintaining or inducing the multi/pluripotency ${ }^{[33]}$. Umbilical cord-derived MSCs, a prototype of fetal MSCs mainly purified from Wharton's jelly, a proteoglycan-rich connective tissue, raise the question whether it is possible to reprogram connective tissue somatic cells to some degree of multipotency/pluripotency by re-establishing a proteoglycan-rich microenvironment. Previous studies show that fibromodulin (FMOD) is a vital component for the maintenance of endogenous stem cell niches ${ }^{[34]}$. In addition, FMOD's essential function in scarless fetal wound healing ${ }^{[35]}$ and reduced scar formation in adult cutaneous wounds (by eliciting a fetal-like phenotype of adult dermal fibroblasts) are suggestive of its potency for cell fate determination and reprogramming ${ }^{[36,37]}$.

In 2012, we successfully generated multipotent cells from human dermal fibroblasts by continuous stimulation with recombinant human FMOD under serum-free conditions at an efficiency of $0.03 \%$ which is comparable to the original retroviral-mediated iPSC reprogramming rates ${ }^{[38,39]}$. Like iPSCs, the yield FMOD reprogrammed (FReP) cells can be 
easily obtained from a skin biopsy and are free from the ethical and logistical constraints that affect the generation of ESCs. Moreover, FReP cells are generated from a protein-based technology without genome integration or oncogene activation. Unlike protein-based iPSC inducing techniques, FMOD reprogramming does not involve the usage of the transcription factors (such as the Yamanaka or Thomson factors) that may induce unwanted gene activation and genomic alterations ${ }^{[23]}$. On the contrary, FMOD reprogramming seems to establish a microenvironment that mimics the quiescent stem cell niche ${ }^{[38]}$. Importantly, unlike undifferentiated iPSCs, including those generated from RNA ${ }^{[40]}$, protein ${ }^{[23]}$ or chemical-based techniques ${ }^{[41]}$, that form teratomas as a consequence of uncontrolled cellular proliferation, undifferentiated FReP cells do not generate tumors in vivo ${ }^{[38]}$. For example, our preliminary data also showed that in an intramuscular microenvironment, iPSCs implantation led to tumor formation in $25 \%$ of tested animals, while no teratomas or other kinds of tumors were generated from $\mathrm{FReP}$ cells in severe combined immunodeficient (SCID) mice ${ }^{[42]}$. Thus, FReP cells closely resemble quiescent stem cells in multiple ways, as expected ${ }^{[38]}$. Interestingly, FReP cells also exhibit several critical characteristics of MUSE cells ${ }^{[26]}$. However, in comparison with MUSE cell collection, FMOD reprogramming does not require hypoxia or low temperatures, and the process is not accompanied by the death of surrounding supporting cells. On the other hand, under osteogenic or myogenic stimulation, FReP cells present faster proliferation and differentiation than iPSCs in vitro ${ }^{[38]}$. Excitingly, our study also showed that FReP cells display a superior capability for bone and skeletal muscle regeneration in comparison with iPSCs ${ }^{[38,43]}$. These results collectively support $\mathrm{FReP}$ cells as a safer cell source for musculoskeletal tissue regeneration than iPSCs ${ }^{[38,43]}$. Nevertheless, it is worth noting that, at the current stage, FMOD reprogramming is a relatively long process and its efficiency is still low; however, this could be significantly improved by a dedicated collaboration focused on refining the reprogramming procedure, similar to what took place with iPSC generation. Moreover, the detailed mechanism of FMOD reprogramming remains to be explored. Clearly, the study of FReP cells is still in the preliminary stage. Further investigation with a broad range of collaboration, particularly long-term safety and efficacy assessments, must be conducted in advance of the clinical applications of FReP cells.

\section{CONCLUSION}

In summary, the advantages and disadvantages of the aforementioned regenerative cell sources are listed in Table 1. In our opinion, although a variety of multi/pluripotent cell sources exhibit potential for use in musculoskeletal regeneration, each of these cell sources has their own obstacles that need to be surmounted before they can be applied on a large scale in clinics. For these reasons, there is still an urgent demand for the development of a safe and easily obtainable cell source for musculoskeletal regeneration.

Table 1. The advantages and disadvantages of different stem cells in musculoskeletal regeneration.

Cell type Advantages Disadvantages

Adult mesenchymal stem cells(MSCs)

1.The most studied stem cell

2.Multipotent (controversial)

3. No ethical issues

Fetal mesenchymal stem cells(MSCs)

\section{Multipotent}

2. Low tumorigenicity

3. No ethical issues

4. No invasive and painful isolation procedures
1. Invasive and painful isolation procedures

2. Insufficient cell yield

3. Tumorigenicity

4. Chromosomal aberrations during cultivation

5. The active mode and stemness are questionable

1. Immunogenicity for allogeneic usage

2. The absence of standard isolation/purification methods

3. Costly storage for autologous application 
Table 1. Cont.

\begin{tabular}{|c|c|c|}
\hline Cell type & Advantages & Disadvantages \\
\hline $\begin{array}{l}\text { Embryonic stem } \\
\text { cells(ESCs) }\end{array}$ & 1. Pluripotent & $\begin{array}{l}\text { 1. Ethical issues } \\
\text { 2. Controversial immunogenicity } \\
\text { 3. Genomic instability and tumorigenicity }\end{array}$ \\
\hline $\begin{array}{l}\text { Induced pluripotent } \\
\text { stem cells(iPSCs) }\end{array}$ & $\begin{array}{l}\text { 1. Obtain and expand easily } \\
\text { 2. Pluripotent } \\
\text { 3. No ethical issues } \\
\text { 4. No immunogenicity }\end{array}$ & $\begin{array}{l}\text { 1. Gene activation and genomic alterations } \\
\text { 2. High tumorigenicity }\end{array}$ \\
\hline $\begin{array}{l}\text { Multi-lineage } \\
\text { differentiating stress } \\
\text { enduring(MUSE) cells }\end{array}$ & $\begin{array}{l}\text { 1. Multipotent/Pluripotent } \\
\text { 2. No ethical issues } \\
\text { 3. No tumorigenicity } \\
\text { (short-term study) }\end{array}$ & $\begin{array}{l}\text { 1. Relative new and lacking of long-term } \\
\text { investigations } \\
\text { 2. Severe cellular stress needed to get enough yield } \\
\text { 3. Share some properties of MSCs (as a } \\
\text { subpopulation of MSCs) } \\
\text { 4. No direct evaluation for bone or muscle } \\
\text { regeneration }\end{array}$ \\
\hline $\begin{array}{l}\text { FMOD reprogrammed } \\
(\mathrm{FReP}) \text { cells }\end{array}$ & $\begin{array}{l}\text { 1. Multipotent } \\
\text { 2. No invasive and painful } \\
\text { isolation procedures } \\
\text { 3. No ethical issues } \\
\text { 4. No genome integration or } \\
\text { oncogene activation } \\
\text { 5. No tumorigenicity } \\
\text { (short-term study) } \\
\text { 6. Superior for bone and } \\
\text { skeletal muscle regeneration }\end{array}$ & $\begin{array}{l}\text { 1. New and short on collaboration } \\
\text { 2. A relatively long reprogramming period } \\
\text { 3. Mechanism is unclear }\end{array}$ \\
\hline
\end{tabular}

\section{CONFLICTS OF INTERESTS}

$Z$. Z. is one of the inventors on fibromodulin-related patents assigned to UCLA. Z.Z. is also a founder and a former officer of Scarless Laboratories, Inc., which sublicenses fibromodulin-related patents from the UC Regents, who also hold equity in the company.

\section{AUTHOR CONTRIBUTIONS}

Z.Z. conceived the presented idea. X.P. wrote the manuscript with support from C.L. and Z.Z.. N.B. modified the grammar and improved the language. All authors contributed to the final manuscript.

\section{REFERENCES}

1. Horton R. GBD 2010: understanding disease, injury, and risk. Lancet. 2012; 380(9859): 2053-2054.

2. Yelin EH, Cisternas M, Watkins-Castillo SI. Health Care Utilization and Economic Cost: The burden of musculoskeletal diseases in the united states. Rosemont, IL: American Academy of Orthopaedic Surgeon; 2011.

3. Carstairs A, Genever P. Stem cell treatment for musculoskeletal disease. Curr Opin Pharmacol. 2014; 16: 1-6.

4. Garcia-Castro J, Trigueros C, Madrenas J, Perez-Simon J, Rodriguez R, Menendez P. Mesenchymal stem cells and their use as cell replacement therapy and disease modelling tool. J Cell Mol Med. 2008; 12(6b): 2552-2565. 
5. Dhanasekaran M, Indumathi S, Lissa R, Harikrishnan R, Rajkumar J, Sudarsanam D. A comprehensive study on optimization of proliferation and differentiation potency of bone marrow derived mesenchymal stem cells under prolonged culture condition. Cytotechnology. 2013; 65(2): 187-197.

6. De la Fuente R, Bernad A, Garcia-Castro J, Martin MC, Cigudosa JC. Retraction: Spontaneous human adult stem cell transformation. Cancer Res. 2010; 70(16): 6682.

7. Dufrane D. Impact of age on human adipose stem cells for bone tissue engineering. Cell transplant. 2017; 26(9): 1496-504.

8. Yao B, Huang S, Gao D, Xie J, Liu N, Fu X. Ageassociated changes in regenerative capabilities of mesenchymal stem cell: impact on chronic wounds repair. Int Wound J. 2016; 13(6): 1252-1259.

9. Sipp D, Robey PG, Turner L. Clear up this stemcell mess. Nature. 2018; 561(7724): 455-457.

10. Caplan AI. New MSC: MSCs as pericytes are sentinels and gatekeepers. J Orthop Res. 2017; 35(6): 1151-1159.

11. Guimarães-Camboa $N$, Cattaneo $P$, Sun $Y$, Moore-Morris T, Gu Y, Dalton ND, Rockenstein E, Masliah E, Peterson KL, Stallcup WB, Chen J, Evans SM. Pericytes of multiple organs do not behave as mesenchymal stem cells in vivo. Cell Stem Cell. 2017; 20(3): 345-359.e5.

12. Lye KL, Nordin N, Vidyadaran S, Thilakavathy K. Mesenchymal stem cells: From stem cells to sarcomas. Cell Biol Int. 2016; 40(6): 610-618.

13. Omelchenko DO, Rzhaninova AA, Goldshtein DV. Comparative transcriptome pairwise analysis of spontaneously transformed multipotent stromal cells from human adipose tissue. Genetika. 2014; 50(1): 96-104.

14. Akimoto K, Kimura K, Nagano M, Takano S, To'a Salazar G, Yamashita T, Ohneda O. Umbilical cord blood-derived mesenchymal stem cells inhibit, but adipose tissue-derived mesenchymal stem cells promote, glioblastoma multiforme proliferation. Stem Cells Dev. 2012; 22(9): 1370-1386.

15. Ren G, Zhao X, Wang Y, Zhang X, Chen X, Xu C, Yuan ZR, Roberts Al, Zhang L, Zheng B, Wen T, Han Y, Rabson AB, Tischfield JA, Shao C, Shi Y. CCR2-dependent recruitment of macrophages by tumor-educated mesenchymal stromal cells promotes tumor development and is mimicked by TNFa. Cell Stem Cell. 2012; 11(6): 812-824.

16. Davies JE, Walker JT, Keating A. Concise Review: Wharton's Jelly: the rich, but enigmatic, source of mesenchymal stromal cells. Stem Cells Transl Med. 2017; 6(7): 1620-1630.
17. Avior Y, Sagi I, Benvenisty N. Pluripotent stem cells in disease modelling and drug discovery. Nat Rev Mol Cell Biol. 2016; 17(3): 170.

18. Sugarman J, Siegel A. How to determine whether existing human embryonic stem cell lines can be used ethically. Cell Stem Cell. 2008; 3(3): 238-239.

19. Araki R, Uda M, Hoki $Y$, Sunayama M, Nakamura M, Ando S, Sugiura M, Ideno H, Shimada A, Nifuji A, Abe M. Negligible immunogenicity of terminally differentiated cells derived from induced pluripotent or embryonic stem cells. Nature. 2013; 494(7435): 100.

20. Koch CA, Geraldes P, Platt JL. Immunosuppression by embryonic stem cells. Stem Cells. 2008; 26(1): 89-98.

21. Lowry W, Richter L, Yachechko R, Pyle A, Tchieu J, Sridharan R, Clark AT, Plath K. Generation of human induced pluripotent stem cells from dermal fibroblasts. Proc Natl Acad Sci. 2008; 105(8): 2883-2888.

22. Phillips MD, Kuznetsov SA, Cherman N, Park K, Chen KG, McClendon BN, Hamilton RS, McKay RD, Chenoweth JG, Mallon BS, Robey $P G$. Directed differentiation of human induced pluripotent stem cells toward bone and cartilage: in vitro versus in vivo assays. Stem Cells Transl Med. 2014; 3(7): 867-878.

23. Furno EL, van der Laan S, Maiorano D. Genomic Instability of Pluripotent Stem Cells: Origin and Consequences. London: Pluripotent Stem Cells-From the Bench to the Clinic; 2016.

24. Kuroda Y, Kitada M, Wakao S, Nishikawa K, Tanimura $\mathrm{Y}$, Makinoshima $\mathrm{H}$, Goda M, Akashi $\mathrm{H}$, Inutsuka A, Niwa A, Shigemoto T, Nabeshima Y, Nakahata T, Nabeshima Y, Fujiyoshi Y, Dezawa M. Unique multipotent cells in adult human mesenchymal cell populations. Proc Natl Acad Sci U S A. 2010: 107(19): 8639-8643.

25. Wakao S, Kitada M, Kuroda Y, Shigemoto T, Matsuse D, Akashi H, Tanimura Y, Tsuchiyama K, Kikuchi T, Goda M, Nakahata T, Fujiyoshi Y, Dezawa M. Multilineage-differentiating stressenduring (Muse) cells are a primary source of induced pluripotent stem cells in human fibroblasts. Proc Natl Acad Sci U S A. 2011: 108(24): 9875-9880.

26. Fisch SC, Gimeno ML, Phan JD, Simerman AA, Dumesic DA, Perone MJ, Chazenbalk GD. Pluripotent nontumorigenic multilineage differentiating stress enduring cells (Muse cells): a seven-year retrospective. Stem Cell Res Ther. 2017; 8(1): 227. 
27. Heneidi S, Simerman AA, Keller E, Singh P, Li $X$, Dumesic DA, Chazenbalk G. Awakened by cellular stress: isolation and characterization of a novel population of pluripotent stem cells derived from human adipose tissue. PloS One. 2013; 8(6): e64752.

28. Byrne J, Pedersen DA, Clepper LL, Nelson M, Sanger WG, Gokhale S, Wolf DP, Mitalipov SM. Producing primate embryonic stem cells by somatic cell nuclear transfer. Nature. 2007; 450(7169): 497.

29. Cowan CA, Atienza J, Melton DA, Eggan K. Nuclear reprogramming of somatic cells after fusion with human embryonic stem cells. Science. 2005; 309(5739): 1369-1373.

30. Hansis C, Barreto G, Maltry N, Niehrs C. Nuclear reprogramming of human somatic cells by xenopus egg extract requires BRG1. Curr Biol. 2004; 14(16): 1475-1480.

31. Zhu $X Q$, Pan $X H$, Wang $W$, Chen $Q$, Pang $R Q$, Cai XM, Hoffman AR, Hu JF. Transient in vitro epigenetic reprogramming of skin fibroblasts into multipotent cells. Biomaterials. 2010; 31(10): 2779-2787.

32. Taranger CK, Noer A, Sørensen AL, Håkelien $A M$, Boquest AC, Collas P. Induction of dedifferentiation, genomewide transcriptional programming, and epigenetic reprogramming by extracts of carcinoma and embryonic stem cells. Mol Biol Cell. 2005; 16(12): 5719-5735.

33. Moore KA, Lemischka IR. Stem cells and their niches. Science. 2006; 311(5769): 1880-1885.

34. Bi Y, Ehirchiou D, Kilts TM, Inkson CA, Embree MC, Sonoyama W, Li L, Leet AI, Seo BM, Zhang L, Shi S, Young MF. Identification of tendon stem/progenitor cells and the role of the extracellular matrix in their niche. Nat Med. 2007; 13(10): 1219.

35. Zheng Z, Zhang X, Dang C, Beanes S, Chang GX, Chen Y, Li CS, Lee KS, Ting K, Soo C. Fibromodulin is essential for fetal-type scarless cutaneous wound healing. Am J Pathol. 2016; 186(11): 2824-2832.

36. Zheng Z, James AW, Li C, Jiang W, Wang JZ, Chang GX, Lee KS, Chen F, Berthiaume EA,
Chen Y, Pan HC, Chen EC, Li W, Zhao Z, Zhang $X$, Ting $\mathrm{K}$, Soo $\mathrm{C}$. Fibromodulin reduces scar formation in adult cutaneous wounds by eliciting a fetal-like phenotype. Signal Transduct Target Ther. 2017; 2: 17050.

37. Jiang W, Ting K, Lee $S$, Zara JN, Song R, Li C, Chen E, Zhang X, Zhao Z, Soo C, Zheng Z. Fibromodulin reduces scar size and increases scar tensile strength in normal and excessivemechanical-loading porcine cutaneous wounds. J Cell Mol Med. 2018; 22(4): 2510-2513.

38. Zheng $Z$, Jian J, Zhang $X$, Zara JN, Yin W, Chiang M, Liu Y, Wang J, Pang S, Ting K, Soo C. Reprogramming of human fibroblasts into multipotent cells with a single ECM proteoglycan, fibromodulin. Biomaterials. 2012; 33(24): 5821-5831.

39. Yu J, Vodyanik MA, Smuga-Otto K, AntosiewiczBourget J, Frane JL, Tian S, Nie J, Jonsdottir GA, Ruotti V, Stewart R, Slukvin II, Thomson JA. Induced pluripotent stem cell lines derived from human somatic cells. Science. 2007; 318(5858): 1917-1920.

40. Kogut I, McCarthy SM, Pavlova M, Astling DP, Chen X, Jakimenko A, Jones KL, Getahun A, Cambier JC, Pasmooij AMG, Jonkman MF, Roop DR, Bilousova G. High-efficiency RNA-based reprogramming of human primary fibroblasts. Nat Commun. 2018; 9(1): 745.

41. Medvedev S, Shevchenko A, Zakian S. Induced pluripotent stem cells: problems and advantages when applying them in regenerative medicine. Acta Naturae. 2010; 2(2): 18-28.

42. Li CS, Yang P, Ting K, Aghaloo T, Lee S, Zhang Y, Khalilinejad K, Murphy MC, Pan HC, Zhang X, Wu B, Zhou YH, Zhao Z, Zheng Z, Soo C. Fibromodulin reprogrammed cells: A novel cell source for bone regeneration. Biomaterials. 2016; 83: 194-206.

43. Ha P, Li C, Kim JK, Jiang W, Chang GX, Yoon S, Chen E, Zhang X, Ting K, Soo C, Zheng Z. Abstract QS51: Skeletal Muscle Regeneration by Fibromodulin Reprogrammed Cells without Tumorigenic Risks. Plast Reconstr Surg Glob Open. 2018; 6(4 Suppl): 148. 